\title{
Possible controls on Arctic clouds by natural aerosols from long- range transport of biogenic emissions and ozone depletion events
}

Rupert Holzinger ${ }^{1}$, Oliver Eppers ${ }^{2,3}$, Kouji Adachi ${ }^{4}$, Heiko Bozem ${ }^{2}$, Markus Hartmann ${ }^{5, a}$, Andreas Herber $^{6}$, Makoto Koike ${ }^{7}$, Dylan B. Millet ${ }^{8}$, Nobuhiro Moteki ${ }^{7}$, Sho Ohata ${ }^{7, b, c}$, Frank Stratmann ${ }^{5}$, Atsushi Yoshida $^{7, d}$

\footnotetext{
${ }^{1}$ Institute for Marine and Atmospheric Research, Utrecht (IMAU), Utrecht University, The Netherlands

${ }^{2}$ Johannes Gutenberg University of Mainz, Institute for Atmospheric Physics, Mainz, Germany

${ }^{3}$ Particle Chemistry Department, Max Planck Institute for Chemistry, Mainz, Germany
}

$15{ }^{4}$ Department of Atmosphere, Ocean, and Earth System Modeling Research, Meteorological Research Institute, Tsukuba, Japan

${ }^{5}$ Atmospheric Chemistry Department (ACD), Leibniz-Institute for Tropospheric Research (TROPOS), Leipzig, Germany

${ }^{6}$ Alfred Wegener Institute Helmholtz Centre for Polar and Marine Research (AWI), Bremerhaven, Germany

${ }^{7}$ Department of Earth and Planetary Science, Graduate School of Science, The University of Tokyo, Tokyo, Japan

$20{ }^{8}$ University of Minnesota, Saint Paul, MN, USA

${ }^{a}$ now at: Atmospheric Science, Department of Chemistry and Molecular Biology, University of Gothenburg, Gothenburg, Sweden

bnow at: Institute for Space-Earth Environmental Research, Nagoya University, Nagoya, Aichi, Japan

${ }^{c}$ now at: Institute for Advanced Research, Nagoya University, Nagoya, Aichi, Japan

25 dnow at: National Institute of Polar Research, Tokyo, Japan

Correspondence to: Rupert Holzinger (r.holzinger@uu.nl) 
Table S1: Campaign ( $\mathrm{n}=7771)$ mean, median, 25\%, and 75\% percentiles for all detected signals that were above the limit of 35 detection for at least $3 \%$ of all measurements.

\begin{tabular}{|c|c|c|c|c|c|}
\hline $\mathrm{m} / \mathrm{Q}$ & $\begin{array}{l}\text { possible } \\
\text { attribution }\end{array}$ & mean & med & $\mathrm{p} 25$ & $\mathrm{p} 75$ \\
\hline 27.021 & & 2.4 & 1.9 & -6.9 & 11 \\
\hline 33.021 & methanol & 191 & 171 & 130 & 230 \\
\hline 34.019 & methanol & 17 & 15 & -11 & 44 \\
\hline 41.031 & & 51 & 43 & 34 & 52 \\
\hline 42.025 & acetonitrile & 20 & 20 & 9.0 & 31 \\
\hline 43.011 & & 42 & 38 & 28 & 48 \\
\hline 44.989 & acetaldehyde & 354 & 321 & 164 & 487 \\
\hline 45.987 & $\begin{array}{l}\text { NO2+, mostly } \\
\text { PAN }\end{array}$ & 78 & 75 & 59 & 92 \\
\hline 47.013 & formic acid & 29 & 27 & -3.1 & 56 \\
\hline 48.981 & & 0.9 & 0.9 & -2.0 & 3.6 \\
\hline 49.014 & & 0.8 & 0.7 & -2.3 & 3.7 \\
\hline 51.001 & & 1.6 & 1.6 & -1.9 & 5.0 \\
\hline 55.041 & & 18 & 13 & 4.7 & 23 \\
\hline 56.051 & & 2.1 & 1.9 & -0.9 & 4.8 \\
\hline 57.023 & & 4.9 & 1.9 & -2.6 & 6.2 \\
\hline 57.065 & & 39 & 32 & 25 & 41 \\
\hline 58.033 & & 3.7 & 3.4 & 0.7 & 6.1 \\
\hline 59.05 & acetone & 561 & 523 & 434 & 658 \\
\hline 59.99 & & 1.1 & 1.1 & -1.4 & 3.7 \\
\hline 60.049 & acetone & 18 & 18 & 13 & 23 \\
\hline 61.03 & acetic acid & 16 & 15 & 4.7 & 26 \\
\hline 62.03 & & 2.3 & 1.5 & -1.1 & 4.3 \\
\hline 63.009 & DMS & 10 & 8.9 & 1.7 & 16 \\
\hline 63.984 & & 0.9 & 0.9 & -1.9 & 3.6 \\
\hline
\end{tabular}




\begin{tabular}{|c|c|c|c|c|c|}
\hline 65.019 & & 3.2 & 3.1 & 0.7 & 5.7 \\
\hline 66.964 & & 0.7 & 0.7 & -1.4 & 2.8 \\
\hline 67.056 & & 1.5 & 0.7 & -1.4 & 3.0 \\
\hline 69.069 & isoprene & 15 & 12 & 7.3 & 17 \\
\hline 70.078 & & 0.9 & 0.8 & -1.3 & 3.0 \\
\hline 71.053 & & 4.5 & 2.4 & -0.6 & 5.4 \\
\hline 73.062 & MEK & 118 & 105 & 89 & 139 \\
\hline 74.033 & & 4.3 & 4.2 & 1.5 & 6.9 \\
\hline 75.043 & proprionic acid & 9.2 & 8.9 & 5.7 & 12 \\
\hline 76.94 & & 0.3 & 0.3 & -1.7 & 2.2 \\
\hline 77.023 & & 1.2 & 1.1 & -0.8 & 3.2 \\
\hline 79.055 & benzene & 63 & 62 & 57 & 67 \\
\hline 80.059 & & 4.3 & 4.1 & 2.2 & 6.2 \\
\hline 80.991 & & 13 & 13 & 10 & 15 \\
\hline 81.062 & & 3.8 & 2.9 & 0.8 & 4.9 \\
\hline 82.959 & & 6.7 & 6.6 & 4.5 & 8.7 \\
\hline 83.081 & & 7.9 & 6.0 & 3.0 & 9.2 \\
\hline 84.946 & & 1.1 & 1.2 & -0.9 & 3.2 \\
\hline 84.967 & & 0.4 & 0.5 & -1.7 & 2.6 \\
\hline 85.056 & & 1.5 & 0.8 & -1.9 & 3.5 \\
\hline 87.055 & pentanone & 16 & 14 & 10 & 20 \\
\hline 88.064 & & 1.0 & 0.9 & -1.1 & 2.9 \\
\hline 91.043 & & 3.1 & 1.8 & -10.6 & 14 \\
\hline 93.07 & toluene & 10 & 8.4 & 5.7 & 11 \\
\hline 95.044 & & 2.2 & 1.2 & -1.5 & 3.8 \\
\hline 96.998 & & 1.5 & 1.6 & -2.1 & 5.0 \\
\hline 97.094 & & 3.8 & 2.4 & -1.0 & 6.0 \\
\hline 99.019 & & 3.6 & 3.4 & 0.6 & 6.2 \\
\hline 99.076 & & 2.1 & 1.8 & -0.7 & 4.3 \\
\hline 100.947 & & 11 & 11 & 7.6 & 14 \\
\hline
\end{tabular}




\begin{tabular}{|c|c|c|c|c|}
\hline 101.033 & 2.7 & 2.4 & -1.5 & 6.3 \\
\hline 102.948 & 5.8 & 5.9 & 1.2 & 11 \\
\hline 104.948 & 1.5 & 1.4 & -1.0 & 3.9 \\
\hline 105.049 & 1.0 & 0.4 & -1.4 & 2.3 \\
\hline 105.941 & 5.6 & 5.4 & -2.1 & 13 \\
\hline 106.07 & 0.7 & 0.4 & -2.9 & 3.9 \\
\hline 106.946 & 4.5 & 3.6 & -4.1 & 12 \\
\hline 107.047 & 2.4 & 0.4 & -5.6 & 6.6 \\
\hline 107.953 & 8.4 & 7.9 & -10 & 25 \\
\hline 108.072 & 1.9 & 0.2 & -11 & 12 \\
\hline 109.089 & 4.8 & 3.7 & 0.8 & 7.0 \\
\hline 111.113 & 5.6 & 4.5 & 1.5 & 7.7 \\
\hline 112.111 & 0.6 & 0.5 & -1.2 & 2.2 \\
\hline 113.041 & 0.9 & 0.8 & -1.4 & 3.0 \\
\hline 113.112 & 1.1 & 1.0 & $\begin{array}{l}-1.0 \\
\end{array}$ & 3.0 \\
\hline 115.05 & 2.6 & 2.4 & -0.1 & 5.0 \\
\hline 116.911 & 56 & 56 & 52 & 60 \\
\hline 118.928 & 52 & 52 & 47 & 57 \\
\hline 120.085 & 0.6 & 0.2 & -2.5 & 3.3 \\
\hline 120.941 & 16 & 16 & 13 & 19 \\
\hline 121.065 & 4.7 & 1.6 & -1.1 & 4.2 \\
\hline 121.958 & 1.8 & 1.7 & -0.4 & 3.8 \\
\hline 122.091 & 1.4 & 1.1 & -0.6 & 2.8 \\
\hline 122.959 & 2.0 & 2.0 & 0.1 & 3.8 \\
\hline 123.097 & 1.4 & 0.9 & -0.9 & 2.8 \\
\hline 124.957 & 1.8 & 1.6 & -2.0 & 5.4 \\
\hline 125.119 & 1.7 & 1.3 & -0.9 & 3.6 \\
\hline 129.087 & 0.7 & 0.4 & -1.7 & 2.4 \\
\hline 134.944 & 0.7 & 0.7 & -1.0 & 2.4 \\
\hline 135.084 & 3.2 & 1.1 & $\begin{array}{l}-1.1 \\
\end{array}$ & 3.3 \\
\hline
\end{tabular}




\begin{tabular}{|l|l|l|l|l|l|}
\hline 137.123 & monoterpenes & 1.4 & 1.0 & -0.7 & 2.7 \\
\hline 138.119 & & 0.2 & 0.2 & -1.4 & 1.7 \\
\hline 139.129 & nopinone & 0.6 & 0.3 & -1.4 & 2.1 \\
\hline 141.085 & & 0.6 & 0.4 & -1.2 & 2.0 \\
\hline 143.109 & & 1.2 & 0.7 & -1.2 & 2.8 \\
\hline 149.054 & & 1.8 & 1.0 & -1.1 & 3.1 \\
\hline 151.131 & & 0.6 & 0.3 & -1.4 & 2.2 \\
\hline 153.098 & & 0.4 & 0.3 & -1.3 & 1.9 \\
\hline 159.093 & & 1.0 & 0.9 & -1.3 & 3.1 \\
\hline 160.909 & & 0.5 & 0.4 & -1.3 & 2.2 \\
\hline 161.097 & & 1.3 & 0.9 & -1.9 & 3.8 \\
\hline 165.157 & & 0.6 & 0.4 & -1.3 & 2.1 \\
\hline 175.102 & & 0.5 & 0.3 & -1.4 & 2.1 \\
\hline 177.167 & & 1.4 & 1.0 & -1.6 & 3.9 \\
\hline 179.179 & & 0.7 & 0.5 & -1.1 & 2.1 \\
\hline 180.937 & & 1.3 & 1.1 & -0.6 & 2.9 \\
\hline 181.079 & & 0.5 & 0.5 & -1.3 & 2.2 \\
\hline 182.939 & & 1.1 & 1.0 & -0.6 & 2.7 \\
\hline 205.184 & sesquiterpenes & 0.4 & 0.4 & -1.2 & 1.9 \\
\hline 330.171 & & 3.0 & 2.2 & -1.8 & 6.8 \\
\hline 355.07 & D5 & 0.3 & 0.2 & -1.0 & 1.5 \\
\hline 358.201 & & 1.2 & 1.1 & -1.6 & 3.8 \\
\hline & & & & & \\
\hline & & & & & \\
\hline
\end{tabular}




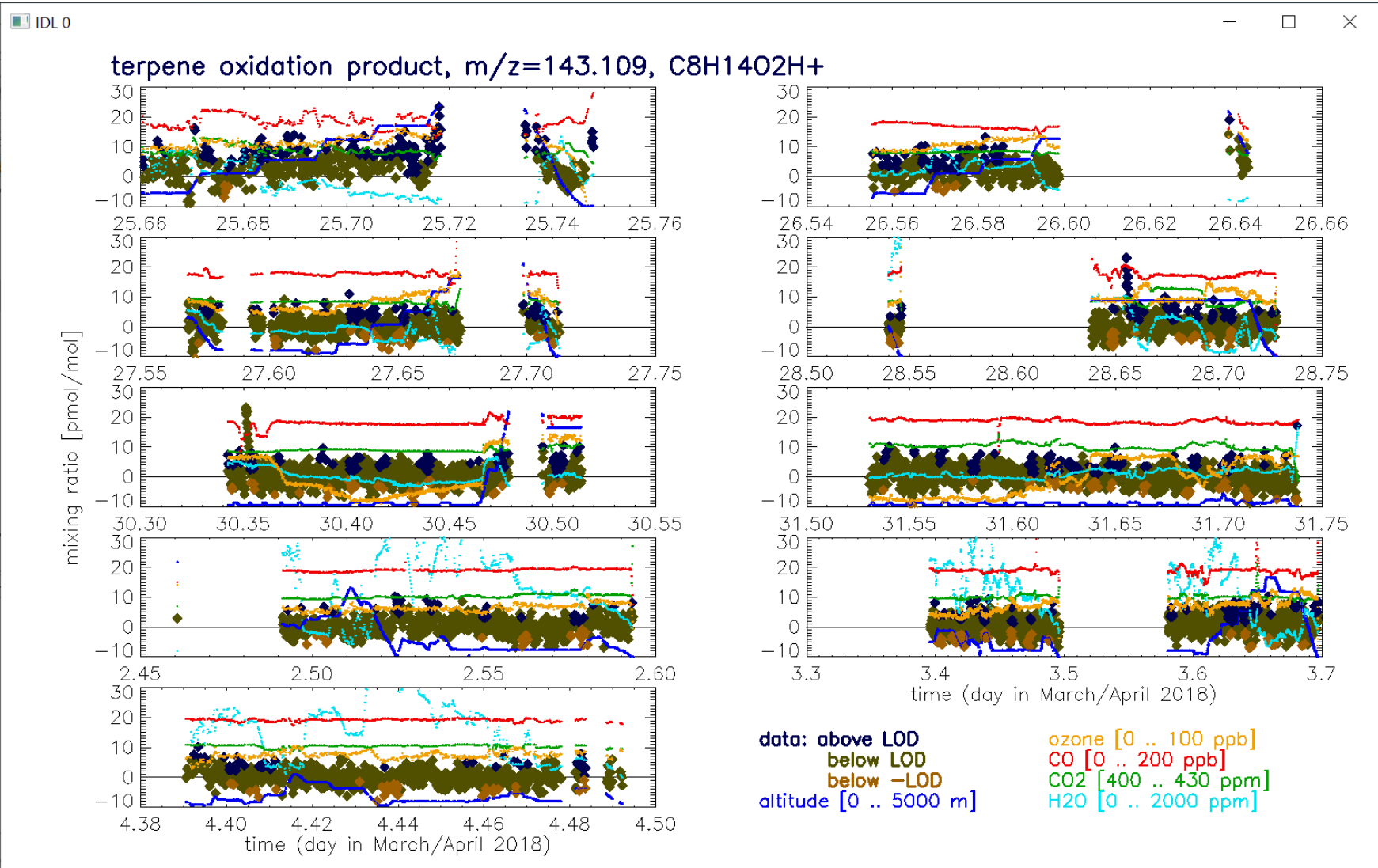

Figure S1: Data and presentation as in Figure 3 but for the signal detected at 143.109 Th. The campaign mean (median) mixing ratio was $1.2(0.7) \mathrm{pmol} / \mathrm{mol}$ for this ion. Measured mixing ratios generally did not emerge above the LOD. The measurements plotted in black

$(n=812)$ were above the LOD, and values plotted in brown $(n=315)$ were negative below -LOD. The fraction above LOD was calculated by subtracting the 'brown' points from the 'black' points and dividing the result by the total number of measurements (n=7771). The result is $6.4 \%$ for this ion. 

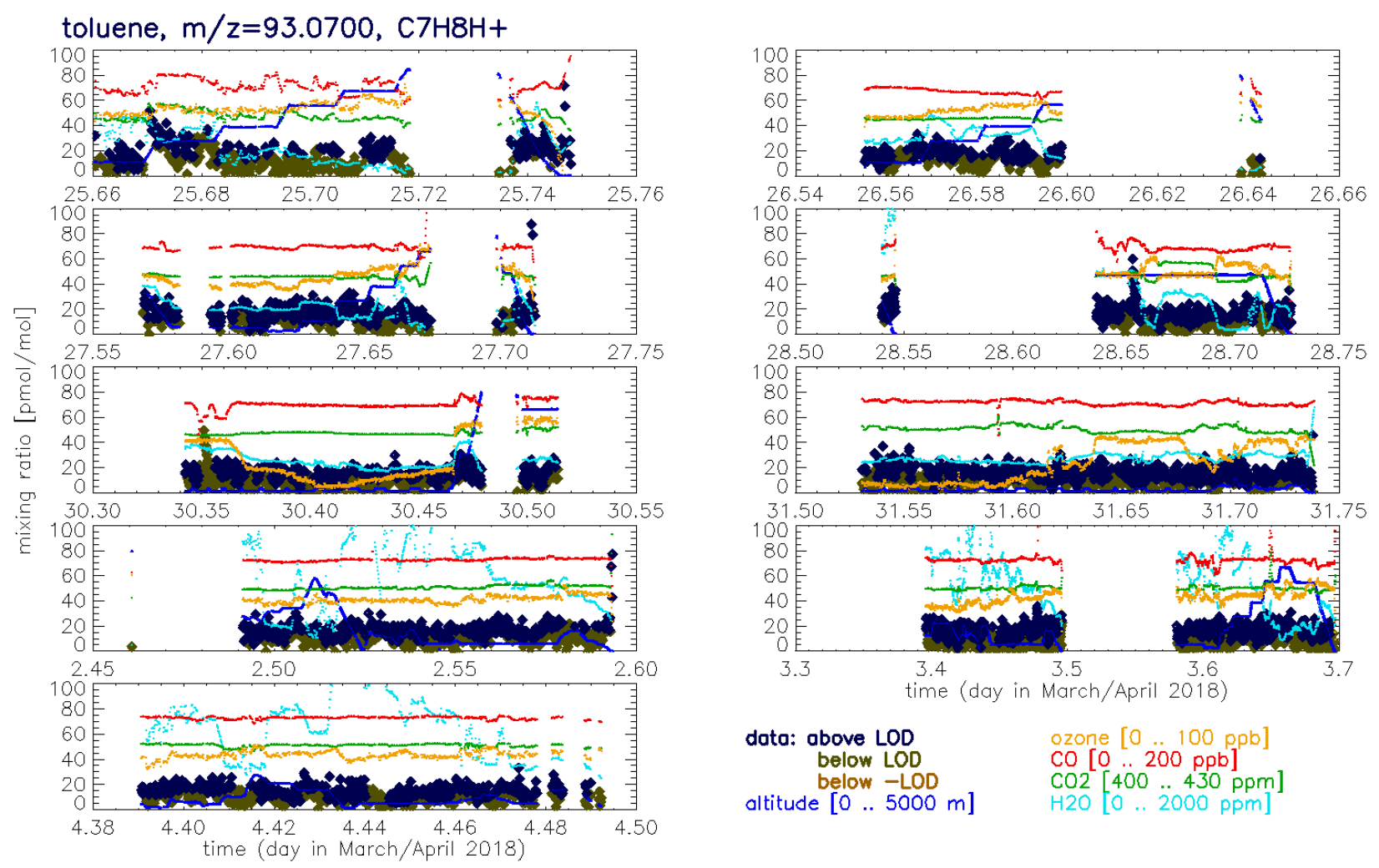
data: above LOD
below LOD

altitude $[0$

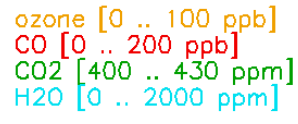

45 Figure S2: Data and presentation as in Figure 3 but for toluene. 

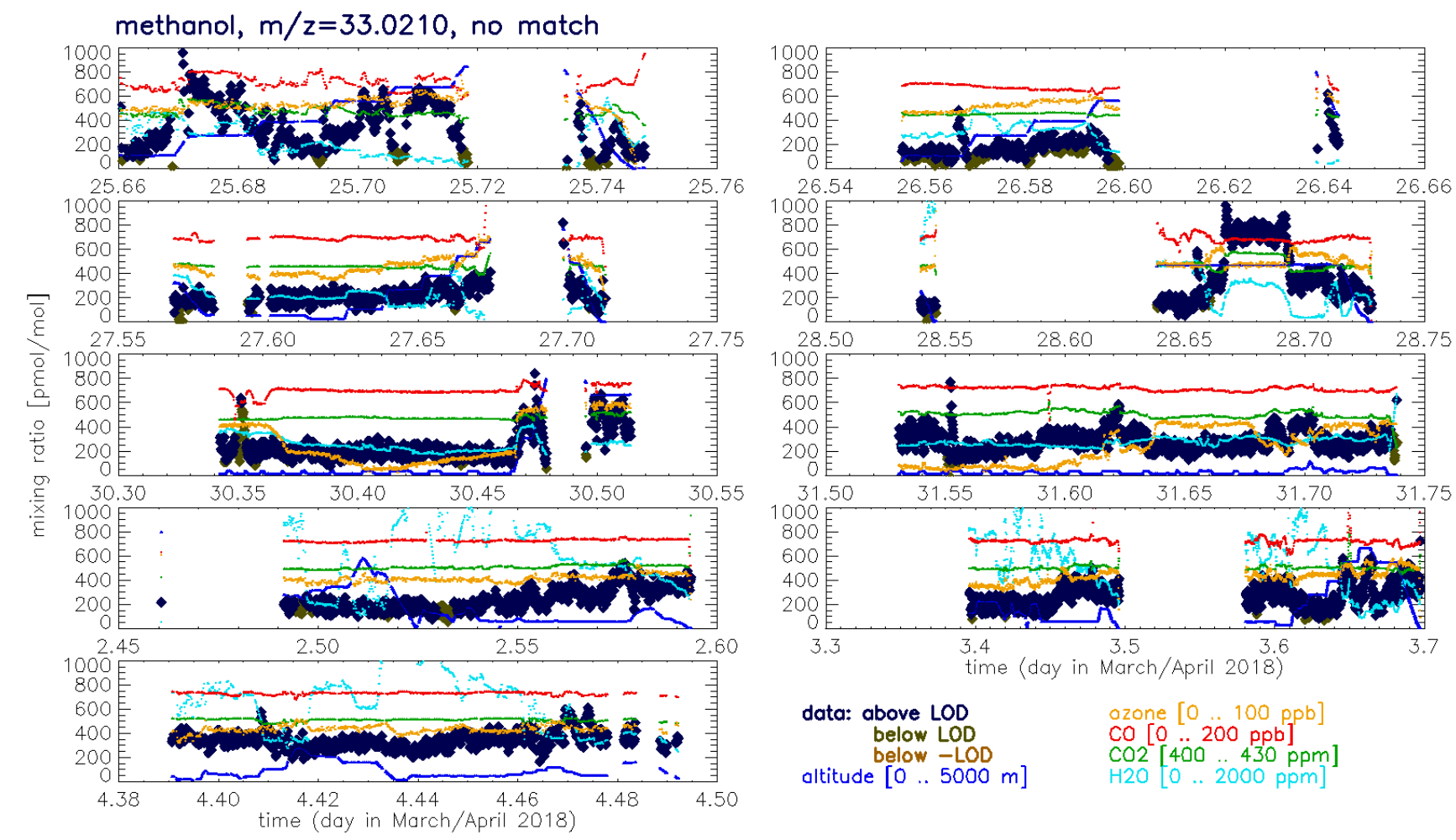

data: above LOD
below LOD

below - LOD

altitude $[0 . .5000 \mathrm{~m}]$

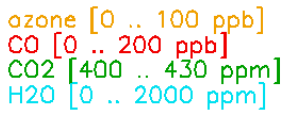

Figure S3: Data and presentation as in Figure 3 but for methanol. 


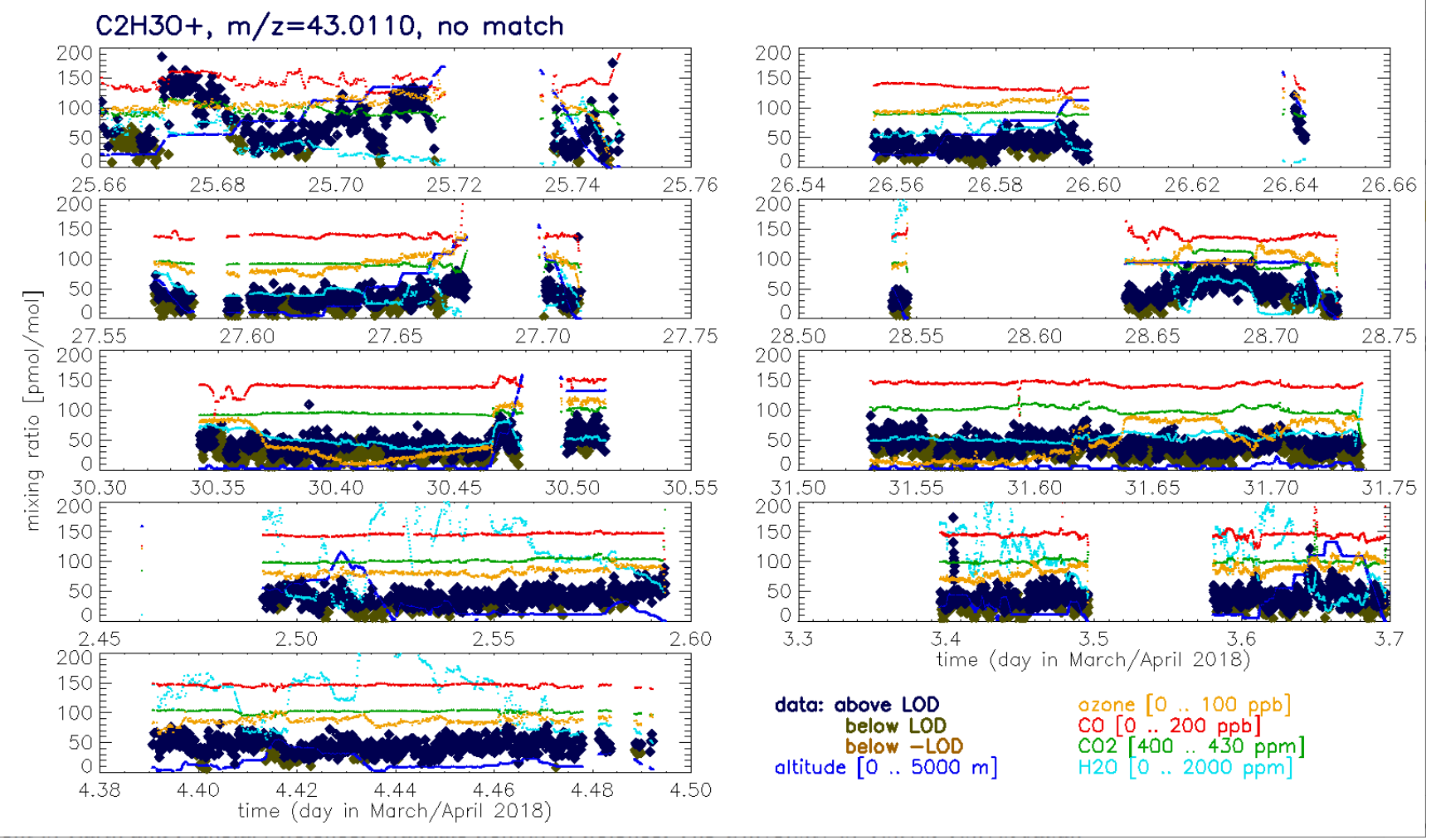

Figure S4: Data and presentation as in Figure 3 but for the signal detected at 43.011 Th. 


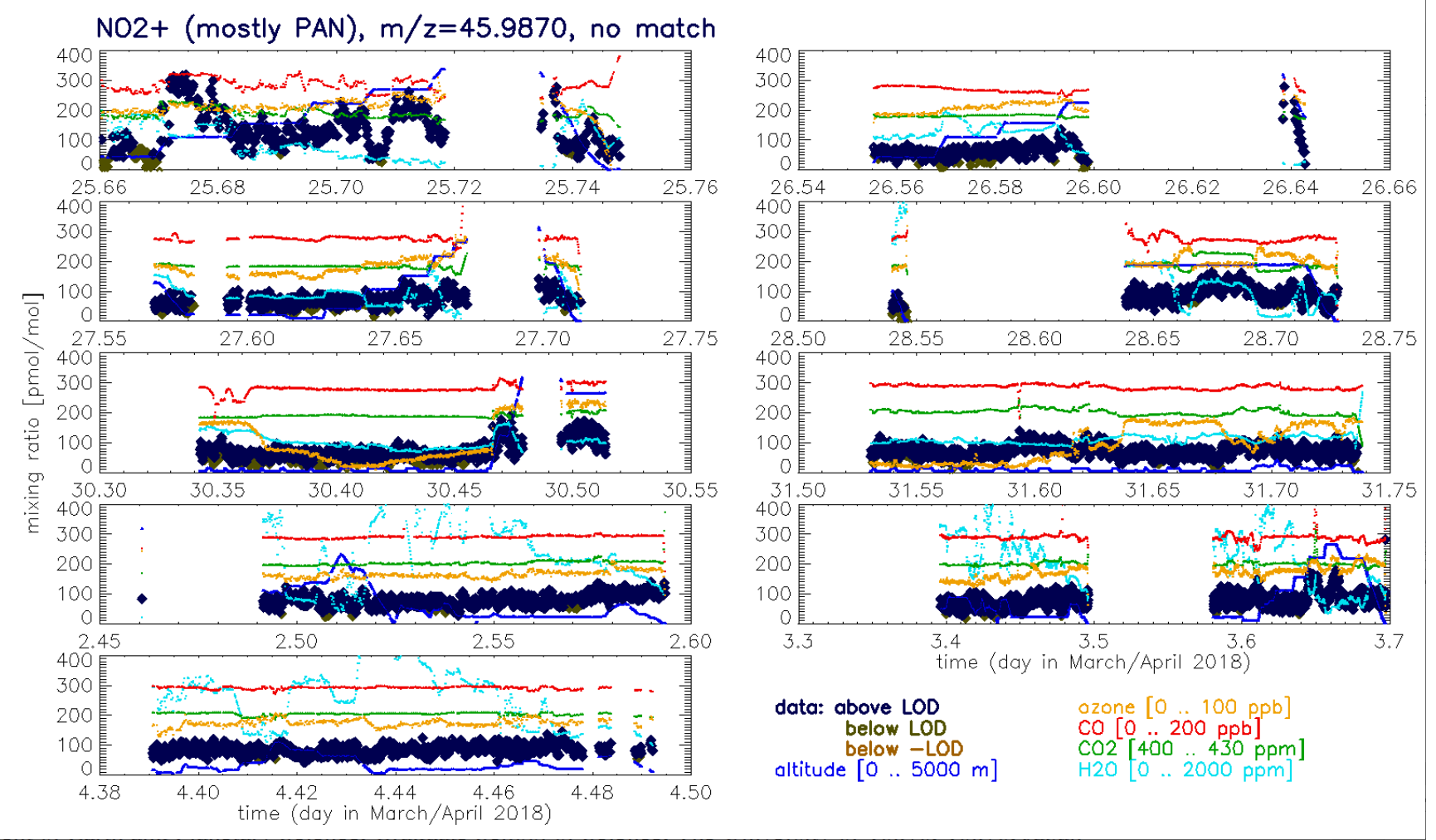

Figure S5: Data and presentation as in Figure 3 but for $\mathrm{NO}_{2}{ }^{+}$(mostly attributed to PAN). 

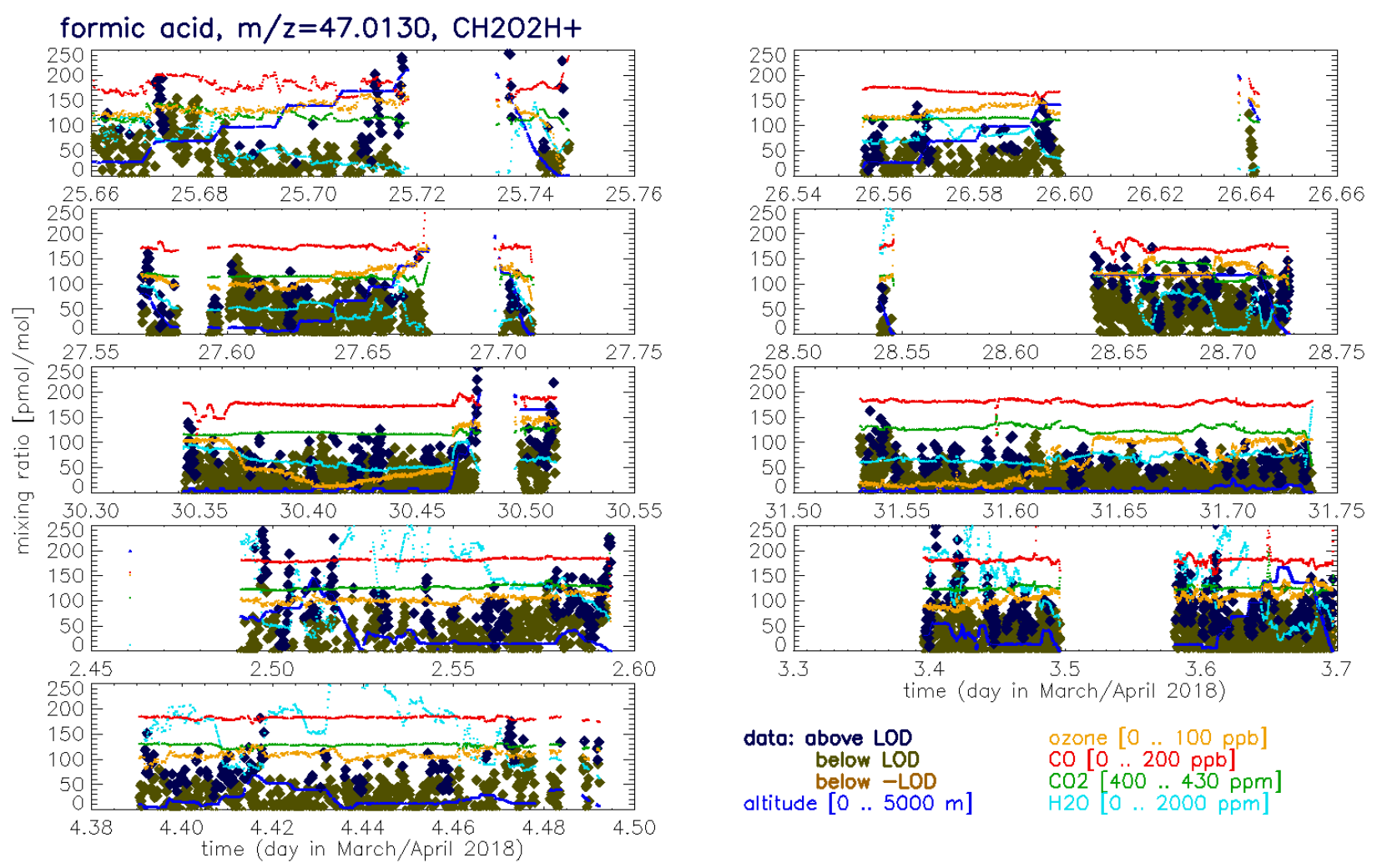

data: above LOD

below LOD

altitude $[0 . .5000 \mathrm{~m}]$

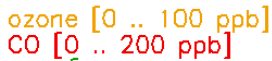

$\mathrm{CO} 2\left[\begin{array}{llll}400 & & 430 & \mathrm{ppm}\end{array}\right]$

Figure S6: Data and presentation as in Figure 3 but for formic acid. 


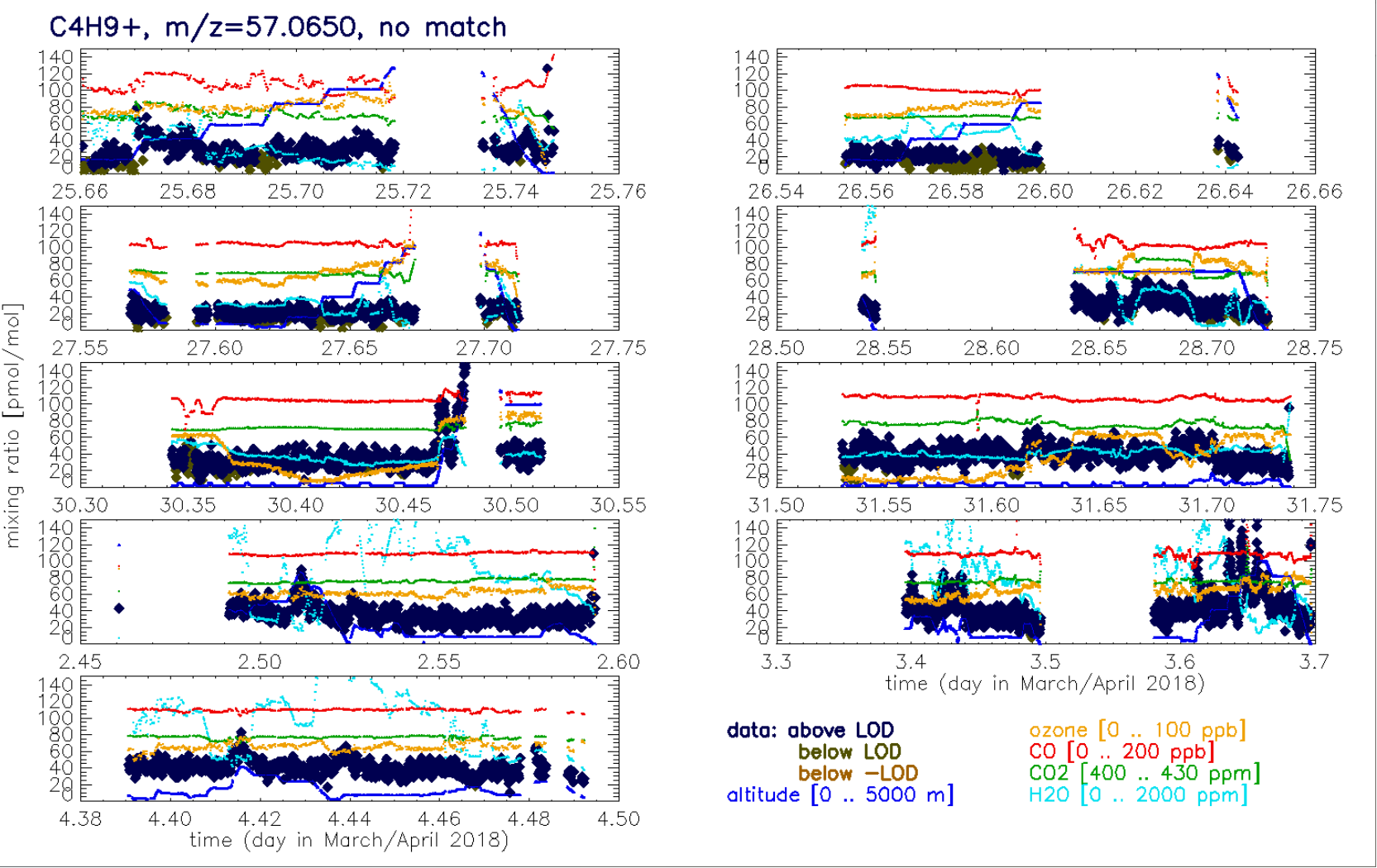

60 Figure S7: Data and presentation as in Figure 3 but for the signal detected at $57.065 \mathrm{Th}$. 
acetic acid, $\mathrm{m} / \mathrm{z}=61.0300, \mathrm{C} 2 \mathrm{H} 4 \mathrm{O} 2 \mathrm{H}+$

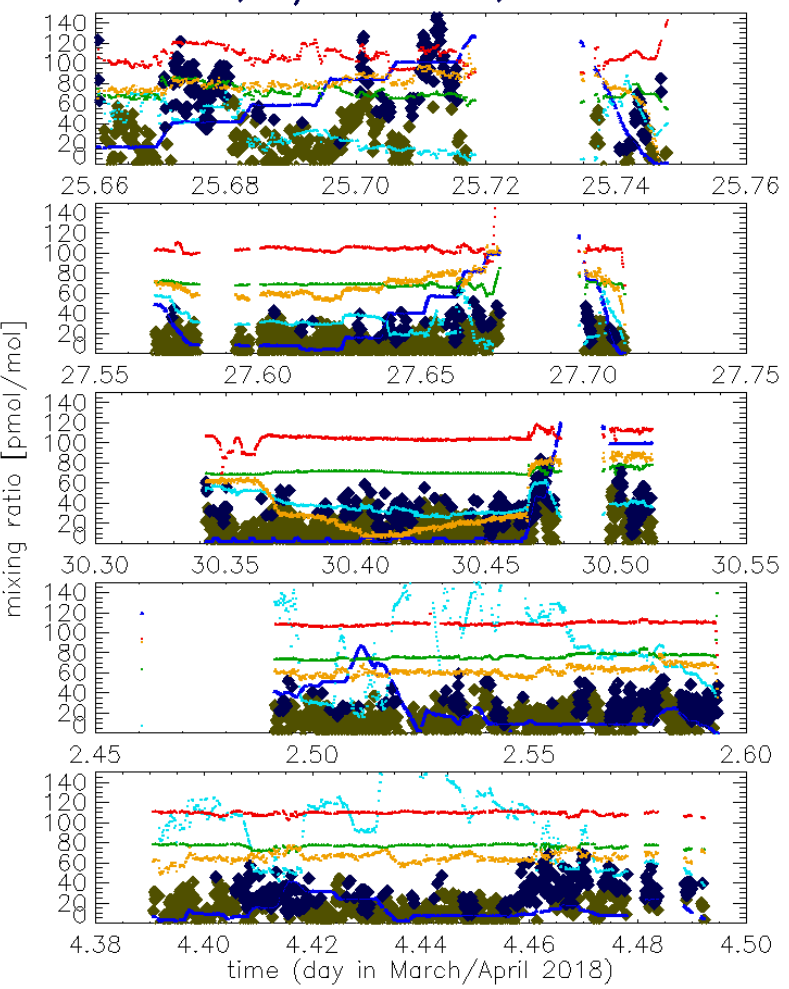

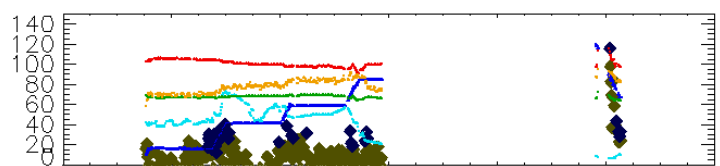

$\begin{array}{lllllll}26.54 & 26.56 & 26.58 & 26.60 & 26.62 & 26.64 & 26.66\end{array}$
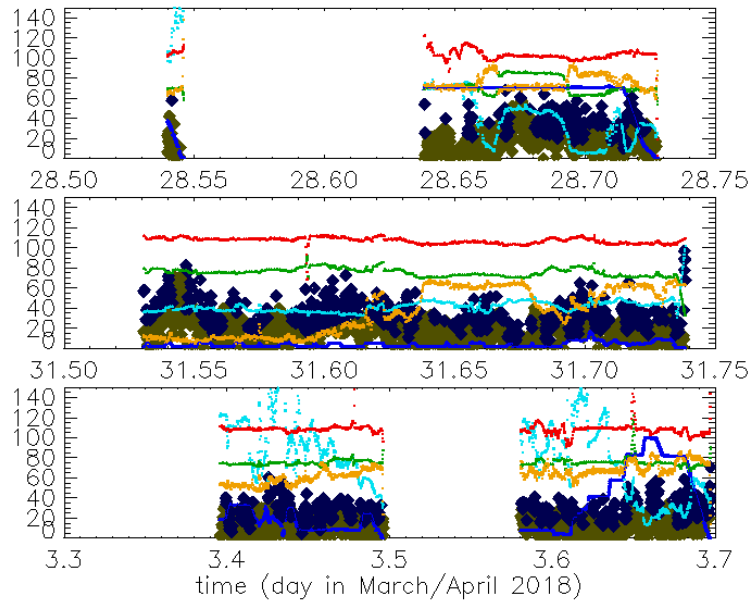

data: above LOD

below LOD

CO $[0 . .200 \mathrm{ppb}]$

$\mathrm{CO} 2[400$.. $430 \mathrm{ppm}]$

altitude $[0$.. $5000 \mathrm{~m}]$

Figure S8: Data and presentation as in Figure 3 but for acetic acid. 


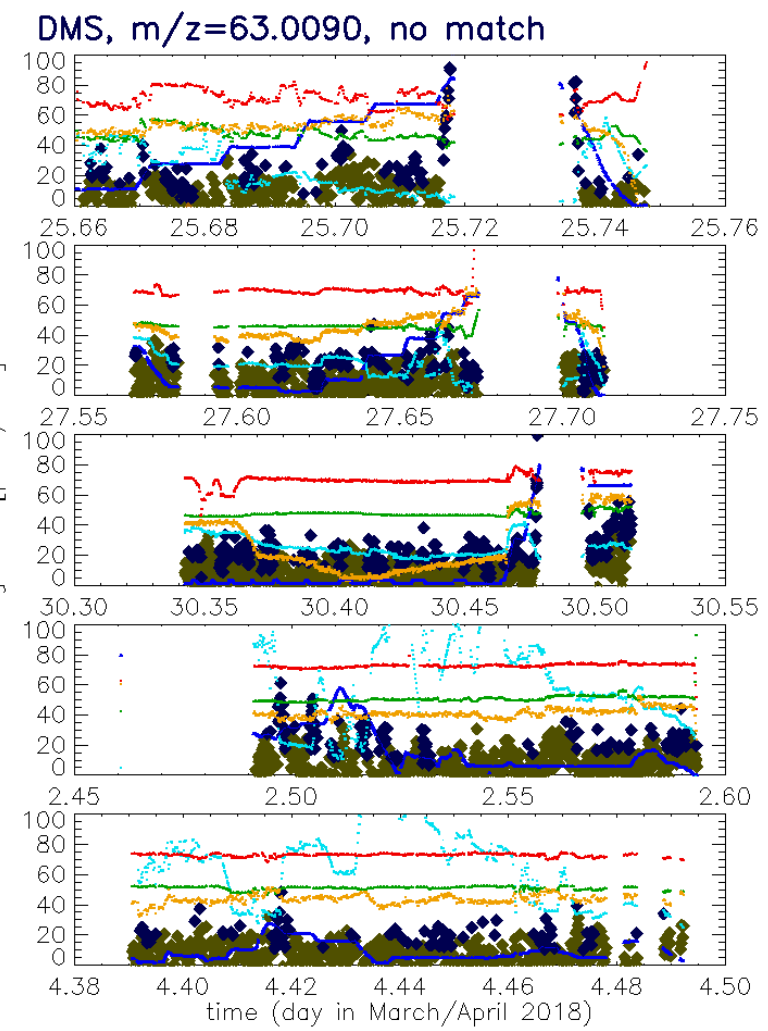

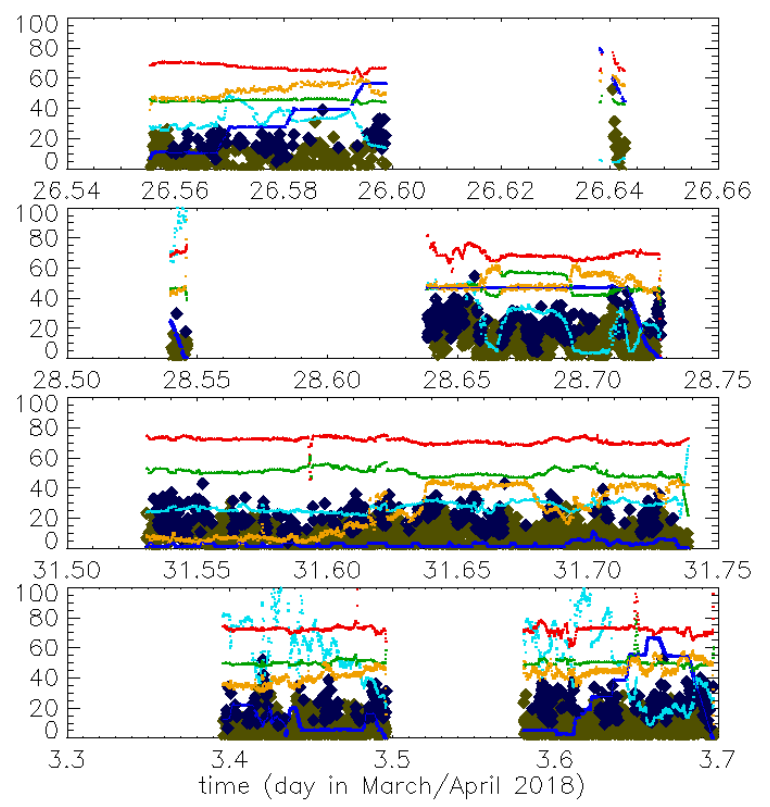

data: above LOD

below LOD

below -LOD

altitude $[0 . .5000 \mathrm{~m}]$

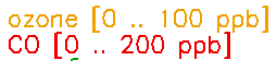

$\mathrm{CO} 2[400$.. $430 \mathrm{ppm}]$

Figure S9: Data and presentation as in Figure 3 but for DMS. 

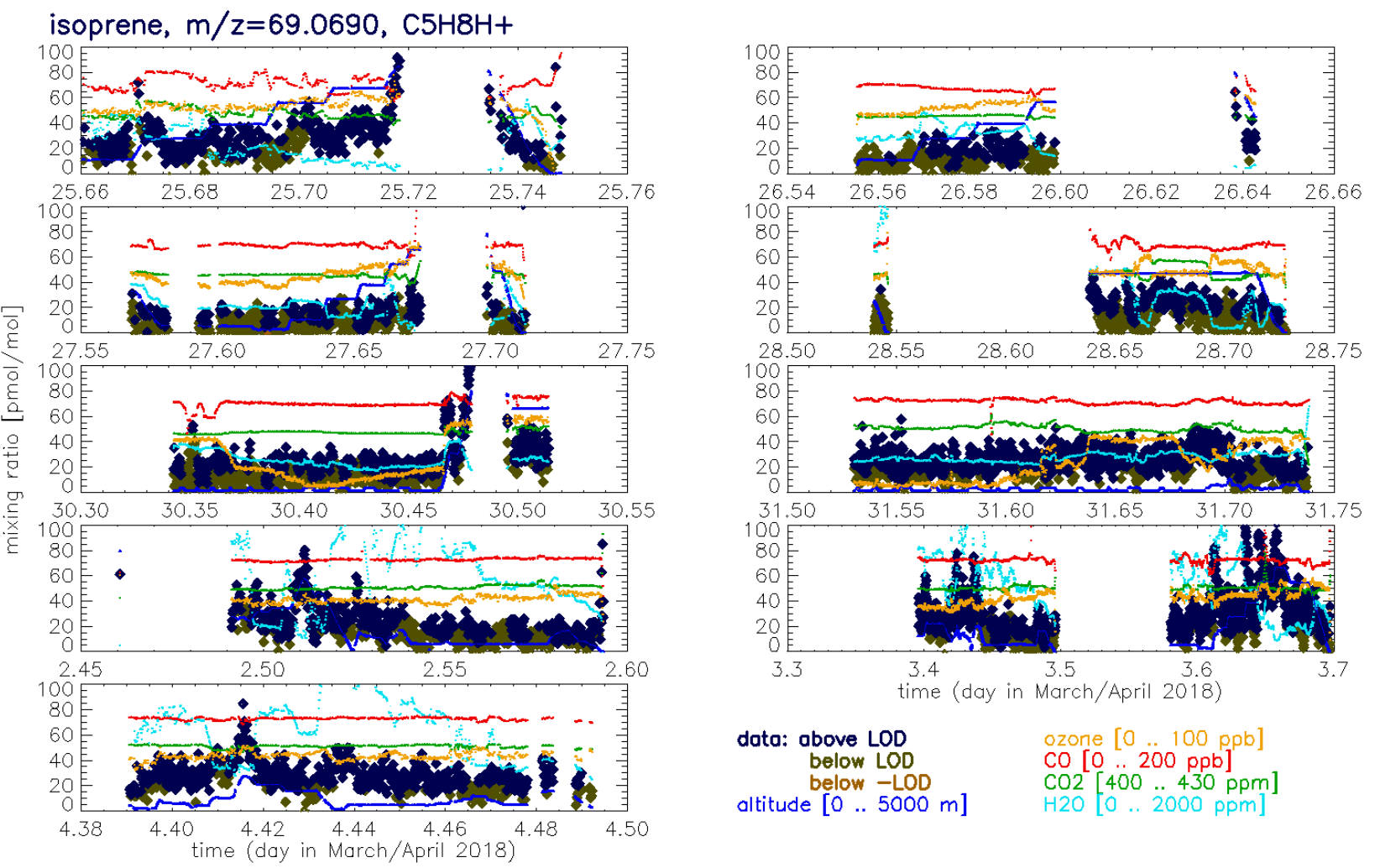

data: above LOD

below LOD

altitude $[0 . .5000 \mathrm{~m}]$

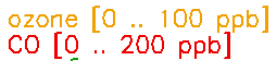

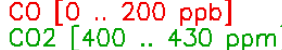

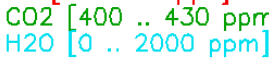

Figure S10: Data and presentation as in Figure 3 but for isoprene. 


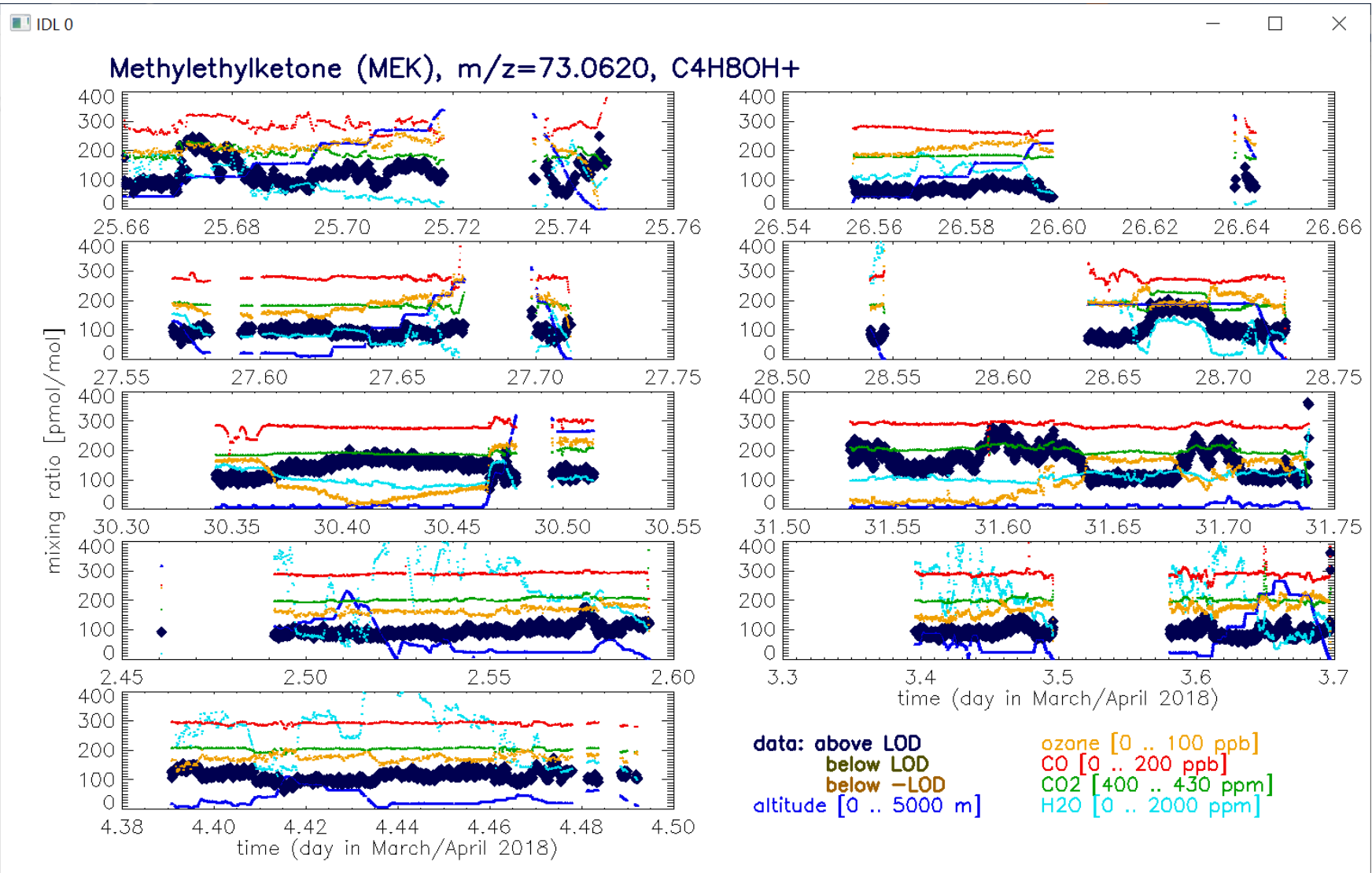

Figure S11: Data and presentation as in Figure 3 but for MEK. 


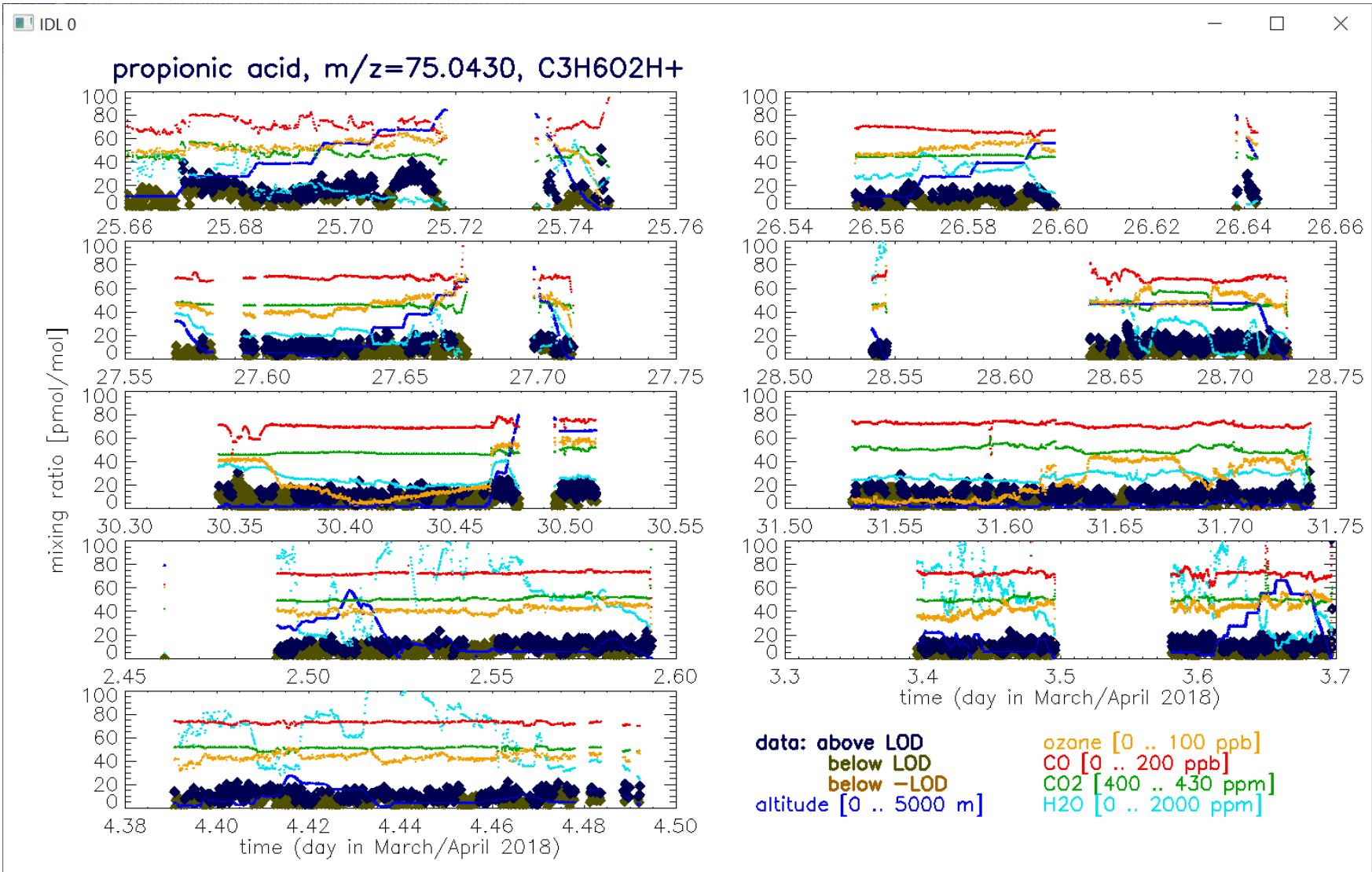

75 Figure S12: Data and presentation as in Figure 3 but for proprionic acid. 

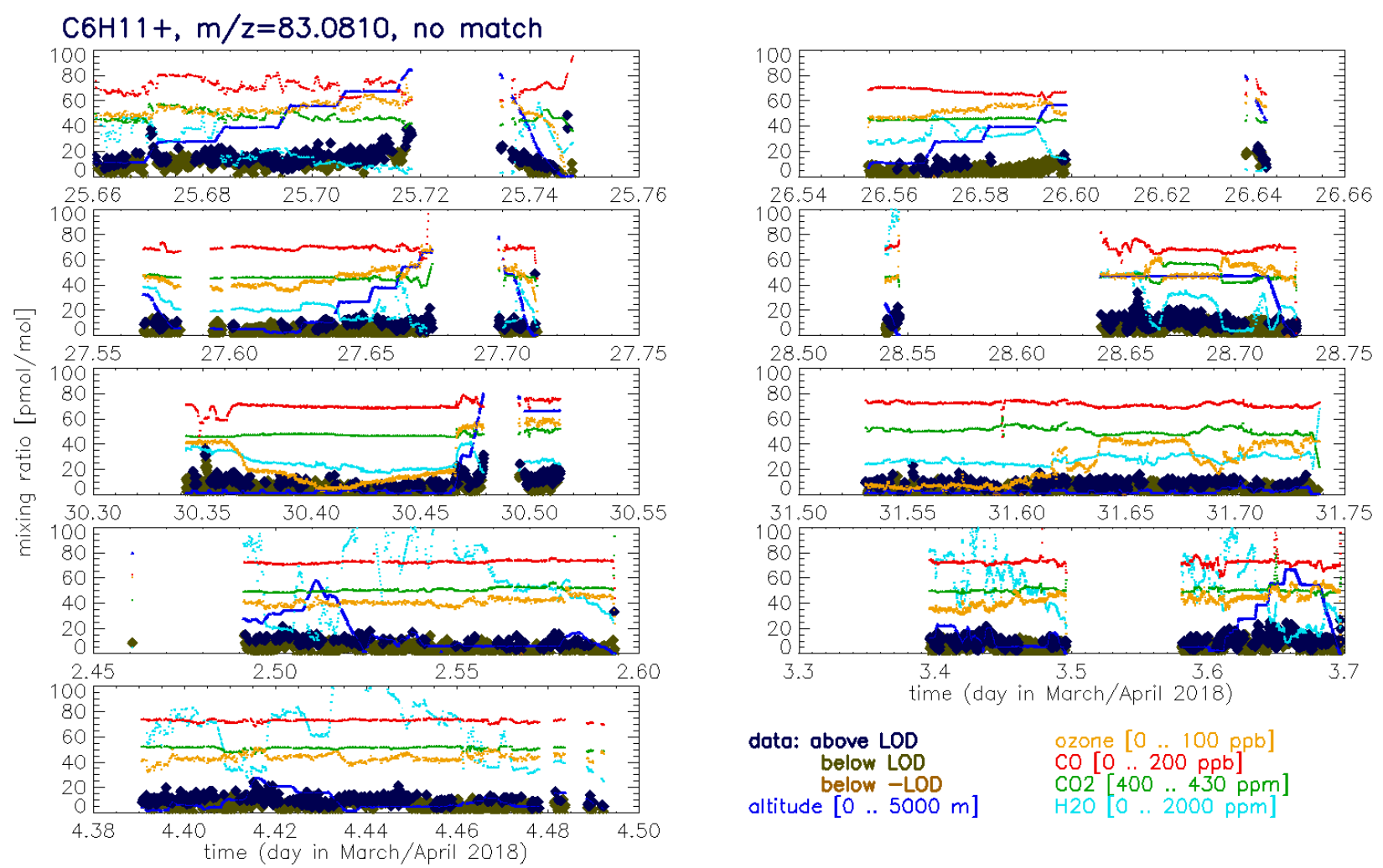
data: above LOD
below LOD
below - LOD
altitude $[0 . .5000 \mathrm{~m}]$
Ozone $[0$
CO $[0.100 \mathrm{ppb}$
$\mathrm{CO} 2[400 . .430 \mathrm{ppm}]$

time (day in March/April 2018)

Figure S13: Data and presentation as in Figure 3 but for the signal detected at $83.081 \mathrm{Th}$. 

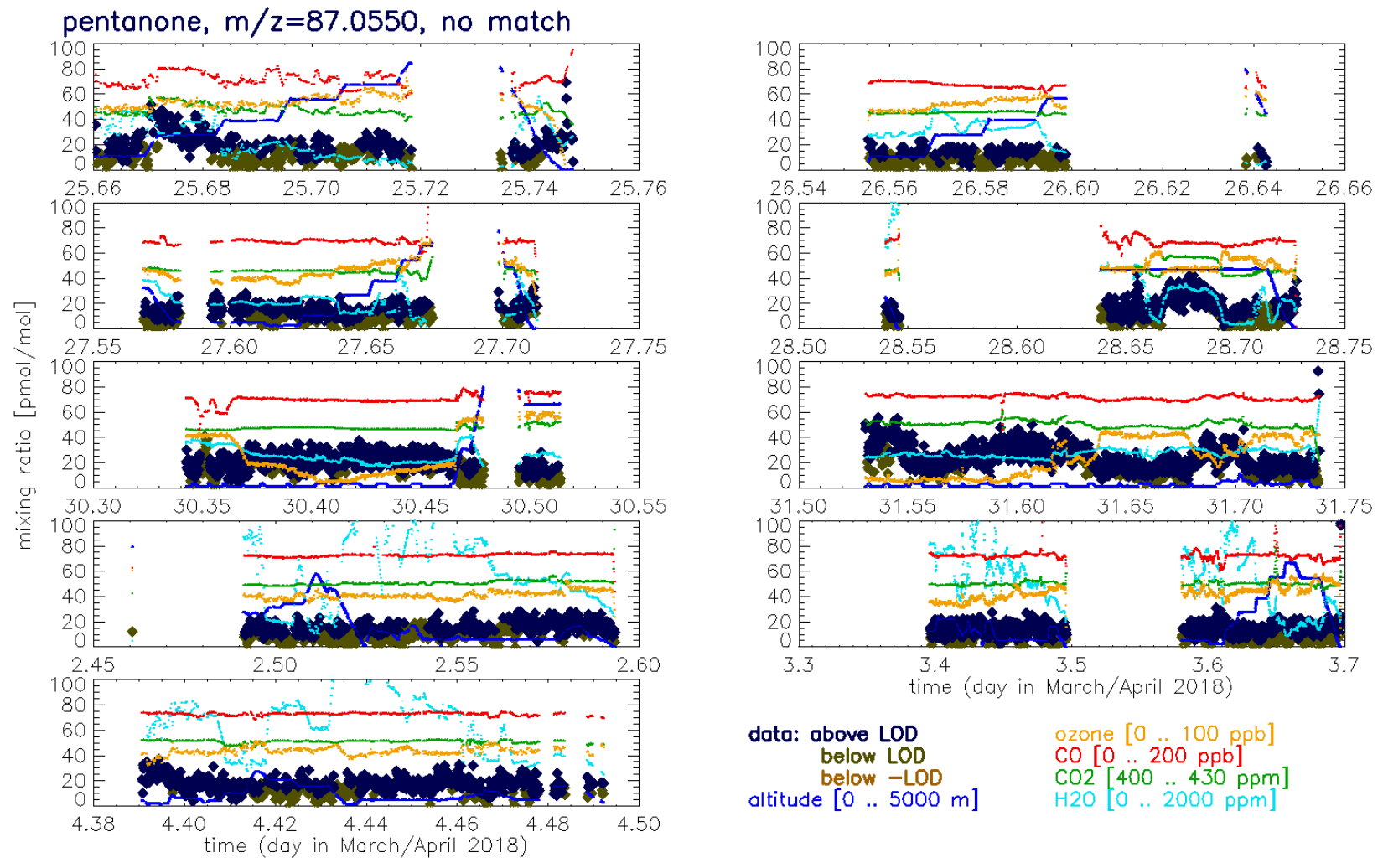

data: above LOD

below LOD

altitude $[0 . .5000 \mathrm{~m}]$
CO $\left[\begin{array}{llll}0 & 200 & 100 & \text { ppp p }\end{array}\right]$

$\mathrm{CO} 2[400$.. $430 \mathrm{ppm}]$

$\mathrm{H} 20[0 \ldots 2000$ ppm]

Figure S14: Data and presentation as in Figure 3 but for pentanone. 